Cinémas

Revue d'études cinématographiques

Journal of Film Studies

Francesco Casetti, Eye of the Century: Film, Experience, Modernity, New York, Columbia University Press, 2008, 288 pp.

\title{
Santiago Hidalgo
}

Volume 21, numéro 1, automne 2010

URI : https://id.erudit.org/iderudit/1005635ar

DOI : https://doi.org/10.7202/1005635ar

Aller au sommaire du numéro

Éditeur(s)

Cinémas

ISSN

1181-6945 (imprimé)

1705-6500 (numérique)

Découvrir la revue

Citer ce compte rendu

Hidalgo, S. (2010). Compte rendu de [Francesco Casetti, Eye of the Century: Film, Experience, Modernity, New York, Columbia University Press, 2008, 288 pp.]

Cinémas, 21(1), 185-203. https://doi.org/10.7202/1005635ar d'utilisation que vous pouvez consulter en ligne.

https://apropos.erudit.org/fr/usagers/politique-dutilisation/ 
Francesco Casetti, Eye of the Century: Film, Experience, Modernity, New York, Columbia University Press, 2008, $288 \mathrm{pp}$.

\section{Introduction}

In his Eye of the Century: Film, Experience and Modernity (originally published in Italian in 2005 as L'occhio del Novecento. Cinema, esperienza, modernità, translated into English by Erin Larkin, with Jennifer Pranolo), Francesco Casetti studies the role cinema played in negotiating the complex experiences of modernity. The first decades of the twentieth century mark the introduction of new urban environments, modes of transportation, communication, industry and social norms which transformed a sense of time, space and self-understanding. While this new environment gave rise to new, exciting opportunities and sensations, it also brought conflicting and disorienting experiences. Casetti argues that cinema filters these experiences and reproduces them in ways that reconcile the contradictory effects, constructing a negotiating "gaze" that the spectator appropriates and employs in his or her own ordinary life. A complex synthesis of theory, history and expressive prose, Eye of the Century offers a unique experience, mirroring in some ways the subject of the book; at times it disorients as much as it clarifies, producing a challenging readership position that evokes the early encounter between audience, cinema and modernity. It is a work that requires self-reflection, interpretation and significant attention to appreciate fully, but which inevitably leads to a novel understanding of the first decades of cinema and the exciting possibilities for film scholarship. 


\section{Structure and Method}

Although Eye of the Century includes material from the first six decades of the twentieth century, special attention is accorded to the 1920s, given their status as a transitional period between a moment of "utopian euphoria" about cinema and its "subsequent systematization" (p. 11). The debate surrounding cinema and modernity in this era became distilled through a number of important authors across Europe and the United States. Several of cinema's attributes justify its unique place in modernity, which Casetti introduces in the opening chapter, "The Gaze of Its Age." Casetti finds in cinema a form that manages to absorb and represent the multitude of diverse experiences of the time, although this does not necessarily mean film functioned strictly as a passive recorder; rather, Casetti conceives of the relationship between cinema and modernity as a dialectic, back and forth process, exemplified in the key concept of "gaze" constituting the central concern of the study. Aside from this quality of "synchronicity" (being "in tune" with its time), three other features of film are presented as essential: its communicative, accessible dimension (that is, its status as a medium, not just art); the cultural myths represented in film narratives which "reflect the issues of emerging social orders" (p. 3); and, most significantly, its ability to "negotiate" the paradoxes of modernity by uniting "conflicting stimuli in an age torn by strife and dilemma" and then "offering them up in their mundane, yet at times touching and magical, everydayness" (p. 3). Film's "gaze" incorporates each of these three features of cinema, though the latter function, as "negotiator," emerges as the most essential in Casetti's argument.

In fact, there is not one, but five "gazes" — partial, composite, penetrating, excited and immersive-that cinema constructs, which can also be spoken of as a single, complex gaze. A chapter is dedicated to each these constituent gazes, the discussion following more or less the same argumentative structure. A fact about modernity, usually in connection with some aspect of cinema, is brought to light, through an array of evidence, including "reviews, analyses, essays, prophecies, political speeches, ironic reporting, drafts of laws, literary pieces and so on" (p. 170). 
Once the network of social discourses "that extend in and around cinema" (p. 170) is charted, illustrating the "synchronicity" between cinema and modernity, Casetti identifies in these discourses, and defines accordingly, salient concepts which are then applied to the analysis of contemporary films. The film analysis, usually of three important films or so per chapter, comprises the largest sections and functions both to illustrate and to counterbalance (although not always successfully) the more theoretical components preceding them. Even taken independently of the argument of which they form a part, the film analysis portions of the book are undoubtedly some of the more exciting in film studies today, especially considering that Casetti revisits films that have already been examined extensively; although he does not reinvent our understanding of these films, he manages to bring a new perspective and attention to forgotten or overlooked details, with an unapologetic poetic flourish that supplies an element of creative performance to the argument.

\section{The Five Gazes}

Examined below are the five film gazes that Casetti presents in the book, whose names follow the chapter titles. Not all gazes receive equal attention, not because of a lack of interest in any one gaze, but rather because some require more explanation. Moreover, the final two gazes serve to illustrate and assess more precisely the logic of the argument, as well as the background theory, so to speak, connecting the various claims Casetti makes about spectatorship over the course of the book; therefore, the analysis of these latter two gazes is proportionately longer.

\section{"Framing the World" (Chapter 2)}

In this chapter on the "partial gaze," Casetti draws on the work of Béla Balázs and Henry James, identifying one of the ways in which modernity transformed the act of perception: "If the modern gaze has a typical characteristic, it is precisely that of being a 'worldly gaze,' inescapably embodied and positioned" (p. 31). At the same time as technology extends our capacity to communicate and see the world, other elements of modernity, Casetti argues, such as film, modern art and romantic literature, 
bring an awareness of the singularity of perception and its incarnation within a particular living subject, in which "the observer is only able to gather a small slice of the "human drama'" (p. 30). Casetti defines the historical trajectory of cinema as being in conflict with these two modes of perception: "Should it move in the sphere of partiality, subjectivity and contingency? Or should it aspire to completeness, objectivity and necessity?" (p. 32). The answer begins to reveal the way in which Casetti imagines that film operated as a kind of negotiator: "Film effectively seized this emergent duality of modern vision, assuring a dialogue between the two" (p. 32).

This dialogue is assured in part because several film devices already incorporate a dialogical, paradoxical component: "With the shot, film puts a limited perimeter of vision on screen; but every film-take seeks to restore a striking 'epiphanic' vision of the world" (p. 32). By "epiphanic" vision, Casetti refers to the capacity of the shot to capture an element of reality that the eye cannot "witness" on its own, allowing the viewer to "seize the reality in which we are immersed" (p. 27). The shot limits vision to a particular field, but in the act of concentrating its gaze on a single point, it reveals something about reality that escapes ordinary vision (what Balázs calls the "unnoticed life"). Second, film editing enables the shot to shift from one to multiple points of view, producing the effect of a "kaleidoscopic" vision that simultaneously offers a partial and a global perspective: "through editing, each shot proposes one and one vision only, but the sequencing of shots permits multiple-even ubiquitous - perspectives." These features of cinema are examined in several films, but I will consider only one example here.

The use of the split screen, superimposition and swish pans, along with rapid editing, combine to produce in Abel Gance's Napoléon (1927) a "mode of grasping the world simultaneously from many sides or in many moments," which Casetti likens to the techniques used by Cubist and Futurist painters. In reference to the rapid editing of a battle scene, Casetti observes that these "points of view follow in succession, but quickly enough as to give the impression of blending one into the next," obviously recalling the "kaleidoscopic vision" metaphor mentioned earlier. 
Moreover, "we see the close-up of Bonaparte, but we have no way of lingering upon it, and thus we end up taking it in 'together' with the other fragments making up the battle scene." Editing produces the effect of placing many competing shots in the same conceptual field of vision, rendering the aggregate as a single unit. Casetti concludes that "we are dealing with a filmic gaze that frees us from the limits of the single glance" (p. 35).

\section{"Double Vision" (Chapter 3)}

In "Double Vision," we are presented less with a "perceptual" gaze than with a "conceptual" one. In addition to a partial gaze that offers the experience of grasping the whole and the part, cinema offers a composite gaze, which incorporates various orders of objective and subjective reality, such as those created in the use of first- and third-person point of view shots. According to Casetti, spectators are able to distinguish between subjective and objective fields, and at the same time experience them as a single, unified field. What qualifies as "subjective" and "objective" in Casetti's argument varies and is sometimes difficult to define. In some cases, this distinction is presented as straightforward, such as films that portray both a fantasy (say a dream) and real world. The distinction is more elusive in contemporary discourses about cinema that allude to the idea of subjective and objective realities, however, although these discourses are made somewhat more accessible through the use of film terminology. One of the perceptual "doubles" that Casetti identifies in this chapter is our capacity both to register "immediate data" (which he refers to as "reports"), and simultaneously to form an internal representation of this data (in the imagination or memory). Balázs refers to the latter as an "inner shot" (the subjective component), distinguishing it from the external, objective shot that merely records reality.

Such conceptual distinctions, as Balázs suggests, are uniquely illustrated in film devices; third-person point of view shots operate in this objective sense, while first-person shots represent the inner, subjective shot. Whether produced through such devices as point of view shots, or through narrative sequences that include both reality and fantasy (of which Casetti includes 
flashbacks), the effect is to jar the spectator into becoming aware of the constructed nature of film. Such awareness becomes useful as a means of likewise recognizing the constructed nature of reality, shaped in part by objective "facts" and their "subjective representations" through media, language, rhetoric and the like.

\section{"The Glass Eye" (Chapter 4)}

The penetrating gaze draws heavily on the "camera-eye" metaphor, derived more from the technological nature of cinema than from aesthetic or narratological characteristics. It is a mechanical, detached and lifeless gaze that nevertheless functions as an extension of the human eye. It responds to another paradox of modernity, the emergence of a technological-industrial world that extends human vision but which at the same time transforms, even replaces, the category of "human." Casetti explores the increasingly clouded boundary between machines and humans from the point of view of a fictional work, Luigi Pirandello's The Notebooks of Serafino Gubbio (1916). A camera operator (Serafino) observes that he feels like "a hand that turns the handle." Casetti interprets this to mean "a person so connected to a machine-a camera - that he becomes a mere appendage" (p. 83). The symbiotic relationship between the motion picture camera and the human body (the eye in particular) structures the theoretical discussion of this section, eventually leading to the posing of a series of familiar questions (which give a sense of Casetti's rhetorical, dialogical approach):

Can it be said that what we see on screen is "someone's" perception if it is the mechanical eye that does the perceiving? What is the relationship between this mechanical eye and man? Does it exonerate his real participation, or does it embolden his decisions and actions? Does it contribute to the mechanization of the world, or does it aid in its rehumanization? (p. 87)

Casetti explores these questions by analyzing films that directly invoke the camera-eye metaphor. In The Cameraman (Edward Sedgwick, 1928), the camera operator Buster Keaton is instructed to capture images that show the "veracity" of a given event (where and when something occurred) as well as its rele- 
vance (its significance "with respect to everyday life"). Thus, the penetrating gaze ascribes meaning to reality in the process of recording it. The analysis of Dziga Vertov's The Man With the Movie Camera (1929), an obvious rejoinder to the discussion (especially through the concept of the "kino-eye"), highlights the camera's capacity to speed up and slow down reality and to record it from different spatial points of view (through camera movements, enlargements, angles); thus, not only does the penetrating gaze record and signify, it also "experiments" with new ways of observing reality. Finally, in his analysis of Merian C. Cooper and Ernest B. Schoedsack's King Kong (1933), Casetti makes a parallel but categorically different observation, arguing that film narrative (as opposed to a technological feature of the camera) operates at two levels, analogous to the story of the giant ape; on the one hand, as we know, film narratives produce pleasurable and exciting experiences, drawing us "closer" to the reality portrayed; on the other, narratives also transform reality into a spectacle for consumption (which Casetti defines as an act of "violence"), "distancing" spectators from reality. This latter observation, however, seems to stretch the definition of the penetrating gaze. Although one can certainly see the parallel Casetti is striving for in this latter example, some of the conceptual continuity, which is essential to maintaining a formal coherency in this type of argument, is lost.

\section{"Strong Sensations" (Chapter 5)}

Georg Simmel's observation in a 1903 article that the modern city has led to an "intensification of nervous stimulation" serves as the organizing principle of chapter 5 on the excited gaze. There is also a kind of simplicity in the way the individual and cinema are organized in the field of modernity in this discussion; contrary to other chapters, we can recognize more clearly the relationship between cause and effects. For these reasons, I will examine this chapter in more detail, attempting to isolate the individual components that are logically connected to explain the film gaze.

Simmel's "The Metropolis and Mental Life" (1903) and Siegfried Kracauer's essay "Cult of Distraction: On Berlin's 
Picture Palaces" (1926) detail some characteristics of the fragmented nature of modernity. The ornate architectural style, frenetic pace of transportation, street advertising and visual culture create a field of experience in which a person becomes ungrounded: "In modernity, nothing remains in its place. Nothing is taken for granted. Nothing is unreachable any longer" (p. 114). How does one contend with such an unstable and destabilizing world? Casetti considers four options, two from Simmel and two from Sigmund Freud (taken from Beyond the Pleasure Principle). Fittingly, Simmel recognizes the type of dichotomy Casetti is interested in exploring: on the one hand, such an environment results in people becoming more rational and intelligent (Simmel suggests there arises a "heightened awareness and a predominance of intelligence in metropolitan man"), enabling people to contend with increasingly complex stimulations; on the other, individuals develop an attitude of indifference, which involves a "blunting of discrimination" of the "meaning and different values of things" (such indifference, Simmel explains, is also caused by capitalism and the rendering of every object into a monetary value-an important point Casetti omits from the original text). Indifference serves to diffuse the intensity of the stimulations (the way one would avoid staring directly at a bright light and view it instead from a corner of the eye).

Freud suggests two other alternatives for "coping with the shock" of external stimulation. One is simply by becoming habituated to the repetition of a given stimulation. Freud defines this mechanism as the formation of a "crust" (described as a "cortical layer") that is "able to resist and repel those very stimuli" (in the same way our fingers develop calluses over time when repeatedly gripping objects in certain ways). A second mechanism is the ludic activity observed in babies of repeating sounds and movements that made a "lasting impression." This repetition enables the baby to gain a mastery of the behaviour and diminish its shocking effects. Thus, concludes Casetti, "we have many interconnected modes of coping with shock: the use of practical reason, the assuming of indifference, the formation of an anti-stimulus shield, and the ludic exercise" (p. 116). The 
dialectic Casetti identifies between shocks and defence mechanisms implies a relatively familiar cause and effect relationship-as certain types of stimulation emerge in the environment, various voluntary and involuntary human mechanisms develop in response. Casetti's argument is to cast cinema as a participant in this dialectic, ensuring that the tensions between stimulation and response are negotiated, "but never in a way that makes the spectator feel truly, definitively lost" (p. 116).

Delving a little deeper into cinema's involvement in this process illustrates the utility, perhaps, of framing the analysis in terms of "gaze." A reasonable question that emerges over the course of the book is whether this concept is at all necessary to Casetti's study. Although recognizing that it is no longer fashionable to speak of a "gaze" isolated from other social domains, Casetti offers two justifications in the Introduction for adopting the term: (1) it emphasizes the fact that cinema was foremost an optical experience; and (2) it focuses attention on the importance of talking about film ("in and of itself") in light of the emergence of other fields (such as cultural studies) that incorporate cinema without necessarily attending to film in its specificity. Casetti does not elaborate, at this late juncture of the book, on why this concept as opposed to other related terms, such as "ways of seeing," "vision," "camera-eye," or even concepts unrelated to seeing but which convey a similar idea, is necessary to his study. Neither does it seem like the concept's importance is tied to some previous theory, such as psychoanalysis, in which its meaning is grounded. For now, I leave the particularity of the concept aside in favour of illustrating how the importance of the term is found, in this instance, primarily in its broadness and flexibility.

Its ambiguity is evident in the way Casetti often substitutes, without explanation, other analogous terms in the place of "gaze," such as those already mentioned, as if these terms were equivalent. Without an ability to discriminate between them, there seems to be no overarching feature the concept gaze uniquely offers other than evoking the theme of "seeing." Nevertheless, the concept serves a significant function in another capacity. It allows Casetti to assemble a diverse set of evidence 
and examples, which would otherwise be categorically disconnected from one another, under a single rubric. Consequently, various features of cinema are able to be studied as one class of thing. The concept gaze, at least in this instance, draws attention to the continuities existing between various domains of evidence, loosely connected by, though not limited to, a "seeing" metaphor, rather than being a clearly specific and determining idea. An example from this chapter will illustrate this point.

Consider what exactly constitutes an "excited gaze." One characteristic is "speed," a commonly cited feature of modernity, along with such key terms as "sensation" and "stimulation." Casetti's analysis of D.W. Griffith's Intolerance (1916) serves as a case study for demonstrating the manifold ways in which "speed" intersects with cinema. Thus, Casetti directs our attention to the literal images of speed, the cars and trains that are instrumental to the resolution of the stories in the film. While "speed" implies in this case the speed of the transportation of bodies, it can also refer to the "rapidity of the execution of a task," which is a "performance ability." The representation of the train's speed also refers us, "metonymically," "to the power of all mechanical devices" to "produce even more merchandise in less time" (p. 118), thus symbolically connecting the image of the train to the industrial revolution. Shifting to another metaphor, such images convey the idea of progress moving forward at an increasing velocity. The narrative sequences in which these elements of speed operate magnify their importance and function, such as chase sequences and the "last second rescues." Finally, novel film devices are closely linked to the experience of velocity, such as mobile camera movements, and most especially crosscutting, which is essential to weaving narrative threads operating at various distances and time periods.

These many "faces" of speed considered, Casetti returns to his dominant mode of emplotment, that of charting the paradoxical experiences that speed produces. For one, these experiences of speed are obviously pleasurable and exciting, while nevertheless producing an element of fear. A basic representation of this dichotomy occurs at a narrative level: "the stories advance at breakneck speed. We enjoy it, but we are flying toward tragedy." 
Another paradox occurs at the level of perception, evoking features of the other film gazes already discussed, such as partial and composite gazes, in which we are simultaneously provided with both greater and lesser abilities to grasp reality. While the excitement generated by speed "alters the senses" it also obscures our perception of things, and thus "we can end up losing ourselves" (p. 118). This dual sensation is of course easy to grasp for those who have experienced an amusement ride; nevertheless, Casetti's concern here is metaphorical, abstracting from this physical experience a mental disorientation resulting from an exposure to various dimensions of speed:

It is legitimate then to ask ourselves, beginning with Griffith's film [Intolerance], in what way the cinema's gaze is able to confront the vortex of the modern world. How does it respond to the acceleration of things, deriving its advantages without exposing itself to risks? (p. 119)

Responding to this question allows Casetti to connect rather abstract features of modernity with a concrete manifestation in which cinema plays the crucial role of negotiator. One should emphasize that a question hanging in the background throughout the study is how all of this "works" at some level. How do spectators find in the film experience a means of contending with the tribulations of modernity? In other words, through what specific "channel" does this negotiation take place? Casetti uses the ambiguous concept gaze to tie some of these loose ends together: "In the first place, it takes a gaze able to grasp the mobility of things, to forsake all forms of fixity and contemplation." One device through which the gaze operates is camera movement, "a true catalogue of the ways in which the dynamics of bodies can be exalted." This is because (notice the change of vocabulary in the following passage) the film gaze produced by these camera movements allows one to attend to reality in a way that is unique to cinema, "The cinema-eye is always able to stay on object bodies without losing sight of them" (p. 119). The latter statement is an example of how Casetti regularly substitutes certain vocabularies for others; in this case, "cinema-eye" provides a closer analogue to the way a camera operates than gaze, 
but each seems to chart a similar and overlapping metaphorical space.

One must also consider the paradoxical consequence of tracking individual bodies in motion: "But a gaze measured by speed can never be content to follow one single event: it must be able to operate simultaneously upon a multiplicity of backdrops." Attributing intentionality to the film gaze, and cinema more generally, is one of the odd features of Casetti's style, which does not allow for a comfortable grasp of the object under study. It raises further questions: Who does this gaze belong to- the spectator? The filmmaker? Culture more broadly? Who, or what, exactly, is not "content"? Casetti seems to invoke, in answering these questions, a disembodied force; but this is in some ways incongruent with a methodological approach focused on social actors, although we must allow that this attribution - the appeal to gaze-is somewhat of a conceit, a shorthand for invoking a collective intentionality. It is a gaze that oddly belongs at the same time to everyone and no one in particular. Nevertheless, one reliable feature of the book is Casetti's inevitable return to film analysis as a means of grounding the discussion, in this case contrasting the fixed attention the shot provides with the mobility of attention in the editing. Thus, crosscutting, which "finds its apex in Intolerance," enables us to observe and connect events from different points of view "without ever losing our sense of position" (p. 120).

Moreover, crosscutting does not apply only to a single episode, but rather creates simultaneous relationships between episodes occurring at different historical periods, "achieving a spatial and temporal ubiquity" (p. 120). Early film reviews seem to validate Casetti's assertion that Intolerance produced special psychological effects resulting from its various displays of "speed," including crosscutting, with one commentator noting a "mental exhaustion" in attempting to maintain pace with the film. In the end, viewers are drawn into a "kind of whirlwind" that nevertheless "prevents us from getting lost" (p. 120). Casetti further suggests that "a truly fast gaze, however, must do even more ... it must be able to anticipate events"; thus the "structure of suspense" ("overlapping the process of crosscutting") in Intolerance enables 
viewers to "see someone in danger" but also to "see that someone is trying to save him." The result is that "the cinema-eye is a provident eye: it is so quick that it is a step ahead of things" (p. 120). These glosses of Intolerance's various speed effects (which are likewise, in some ways, "exhausting" for the reader) are eventually summarized in a single, succinct statement describing the excited gaze: "Thus, we have mobility, ubiquity and foresight: the film-eye is able to deal with speed" (p. 120).

Still, at one level we are not satisfied. How does this spectator-film relationship work? Casetti comes back to a possible answer: "This ability to face the challenge of speed-one of the typical obsessions of modernity-is exemplified in Intolerance by the race against time in which the condemned man's wife and friends engage." In experiencing such sequences, we are given to the sense of not being able to keep up, of being too late. However,

The cinematic gaze, as Intolerance illustrates, assures a positive outcome: its capacity to keep pace with events, to jump between places, and to project itself toward the fatal moment, ensures that the eye (and thus, ideally, the observer) arrives at the place of the tragedy before it occurs-perhaps with a wildly beating heart, but certain of rescue (pp. 121-22).

Intolerance is only one of three films Casetti analyzes in order to illustrate an aspect of the "sensory excitement" generated by the "excited gaze." Aside from "the thrill of movement" (in Intolerance), other sensory excitements include "the thrill of change" (in Eisenstein's Old and New, 1926) and "the thrill of rhythm" (in Mervyn Leroy's Gold Diggers of 1933, 1933). Casetti summarizes the "dangers associated with such sensory excitement":

the risk of losing one's way (the world is moving forward, and I don't know where I am); the risk of not understanding new forms (the world is changing, and I do not recognize it, nor do I recognize myself); and the risk of losing the meaning of things (the world is following a rhythm, and I do not understand why) (p. 135). 
Finally, Casetti closes the loop and brings us back to his discussion of Simmel and Freud, arguing that "cinema develops a dual line of behaviour" by putting "into play an excited gaze that gathers stimuli and boosts them," simultaneously giving "this gaze adequate defenses, which protect it from possible dangers" (p. 135). Belonging neither entirely to the film nor to the spectator, the gaze seems to be a mutually constructed event produced by the film experience, or rather, the accumulation of film experiences, which serves to inoculate the spectator from the intense stimulation of modernity.

We have then, in this gloss of Casetti's presentation of the excited gaze, a variety of different rhetorical and descriptive statements that, once assembled, offer one possible interpretation of the underlying structure of his work. We see that "gaze" operates as a poetic, elusive and orienting concept that allows Casetti to examine a number of interrelated ideas under a similar heading; these various features of cinema-editing, camera movements, literal images, metaphors, narrative structures, genres-along with the network of discourses consolidating around these themes, produce an experience that enables the spectator, potentially, to resolve internal conflicts arising from modernity. This process is not necessarily limited to visual phenomena, but rather is an experience incorporating every facet of consciousness-memory, cognition, attention, intention (one important omission, though, is the role of language). The gaze is, in brief, the process of how we become who we are, told through the allegory of cinema, and presented as if limited to the visual field. Certainly, Casetti does not imply this limitation in his argument; it is a limit of the definition of the term itself, as suggested by Casetti in his justification. Therefore, in studying closely the development of the argument, we are learning how Casetti thinks we become social beings. The gaze, as stated, is shorthand for this process, a term which facilitates its future recollection as an analytical concept, associates it directly to the author, focuses attention on certain features of this process, and evokes a history of older discussions of film theory around the notion of gaze. 


\section{"Immersive gaze" (Chapter 6)}

The last chapter on the film gaze offers a logical conclusion to the preceding analyses, which raised questions about the nature of the exchange between the spectator and cinema and the status of the "gaze" in this operation. Casetti's discussion of the "immersive gaze" is more directly about this problem, drawing on reflexive films that foreground spectatorship as potential models, and literature that directly addresses the concept "gaze." It is admittedly difficult to reduce Casetti's subtle analysis of these various texts to axioms, but this is also the outcome of the more general methodological challenge the book presents: that of separating Casetti's theoretical claims from his rhetorical description of them. His theoretical propositions are often embedded in relatively dense, occasionally ambiguous prose and are not necessarily given to a straightforward and logical organization. Following a consideration of comments from Jean Epstein's "The Cinema Seen from Etna" (1926) and Hans Blumenberg's Shipwreck with Spectator: Paradigm of a Metaphor for Existence (1979), in which Casetti argues that modernity reconfigured the relationship between the observer and the observed, creating a spectatorship position "intertwined ... with the object of [one's] gaze," he offers the type of poetic flourish I allude to:

In both cases [Epstein and Blumenberg's metaphors], there is the idea that what modernity brings to light is an ever-closer intimacy with the surrounding universe and, at the same time, the progressive loss of all certainty. All distance is wiped out. Complicities are created. Coordinates are lost. We enter into an unstable world, and we feel unstable as well. The observer is "inside" the observed world, yet with no precise place. He is situated amidst the sea, on the mountain of fire, on the glass stairs. He is at risk, exposed to winds and waves, exposed to the lava, exposed to himself (p. 144).

This is a style of writing that constantly interweaves metaphorical digressions and which uses the voices of the authors it quotes to present evidence, question ideas and establish mood, as in the example above, creating a rather seamless continuity between the various interlocutors. For this reason, it 
is not always clear where Casetti himself stands in relation to external observers; for instance, whether he agrees or not with these ideas: he sometimes plays the role of conductor linking them together and at others intervenes as performer and creator. His is, indeed, a complex prose (which may be partially attributable to the translation from Italian to English); this forms part of the work's intrigue, but does not necessarily allow for an overarching synthetic view of the whole. We are left with the feeling of grasping only fragments of the puzzle and of being carried along by Casetti's assured presentation, with certain synoptic and perfunctory descriptions occasionally disclosing and revealing more substantial regions, as when he unequivocally asserts that

The spectator before the screen tends to connect with what he is watching. He projects himself onto and at the same time identifies with the shown reality. He feels it as living and feels himself living it (p. 143).

Thus, we understand that Casetti is striving to define the nature of the relationship between the spectator and film as primarily one of "connection" and "identification." Certainly, lurking in these characterizations are the remnants of psychoanalysis' powerful effect on film theory, from which the popularization of the "gaze" concept emerged; Casetti generally attempts to maintain a distance from psychoanalysis, however, while occasionally appropriating its terminology, in an effort to refashion a different way of using "gaze" constructively in historical analysis. This is not an easy rhetorical manoeuvre, and one feels the centrifugal force of psychoanalytic discourse often pulling the argument in its direction.

Certainly, the gaze cannot easily be separated from one of its parent discourses, even with the justifications Casetti initially provides. Possibly for this reason, Casetti introduces an antecedent to film theory's appropriation of gaze, in the form of Sartre's Being and Nothingness, in which the concept is perhaps first elaborated. In a brief digression, Sartre's theory of "the look" is examined in relation to Casetti's own reworking of the gaze. In fact, it marks the first moment in Eye of the Century in 
which the reification of "the gaze," as a disembodied construct belonging to no one in particular, is addressed. Following Sartre, Casetti explains the "filmic experience" as a series of overlapping gazes that connect spectators with film, spectators with spectators, and spectators with the world: "it is precisely in front of a screen that we best feel the observed observing, that we feel ourselves inside a gaze that is no longer ours alone, in communion with a world that carries us away" (p. 162). Casetti envisions a gaze that incorporates more than a one-way spectator-film position, as 1970s film theory made manifest in its own appropriation of gaze; he sees it rather as a "game of cross-gazes" in which the individual partakes in a multiplicity of gazes—one's own, one's neighbour's in the theatre, the gaze of the film, the gaze of the agents in the film, the gaze of those who produced the film, and, these collectively, as a reality that belongs to no one in particular, yet still acts on the individual. As Casetti suggests, spectators "enter into a relationship with another reality, and this allows them to acquire a real knowledge, though it is through the onscreen illusions" (p. 147). It is a difficult conceptualization to grasp, yet the existence of such interplay in the field of gazes is essential to Casetti's argument.

Nevertheless, Casetti always returns to film material that is familiar and intuitive. The "immersive" gaze essentially has three features, each marked by, though not limited to, a particular cinematic device: it brings the spectator close to the screen, such as in the close-up; it situates the spectator, through the craneshot, in relation to other spectators and to the environment (as analogously represented in The Crowd [King Vidor, 1928], when an individual defines himself in relation to a modern city crowd); it conflates our subjectivity with the subjectivities displayed on screen through the appropriately named semi-subjective shot, in which both the object of the gaze and the holder of the gaze are simultaneously in the same shot, achieving a "unity between observer and the observed" extending to the spectator (p. 157). Thus, "[film's] position is sealed by its capacity to offer itself as a field of cross-gazes that include and embrace the observer, the observed and the situation" (p. 165). 


\section{Conclusion}

In an unconventional move, Casetti presents, at least in part, an explanation of his methodology in the final chapter, "Glosses, Oxymorons, and Discipline." I have already mentioned his approach of charting the network of social discourses "that extend in and around" cinema (p. 170). Once these discourses are compiled, none of which necessarily holds any authority over the others, they are combined and matched with the discourses generated by film; this, in turn, creates "glosses for the cinematographic phenomenon that help, if not directly determine, its intelligence and understanding" (p. 170). This interchange between cinema and the network of discourses surrounding it constitutes Casetti's field of study, in which the spectator obviously plays a decisive and instrumental role. The material result of these exchanges, which the spectator receives and negotiates, is "emblems" manifested "in some exemplary themes, as well as through certain devices of language and apparatus."

Casetti displays in this final chapter a profound awareness of the method and implications of his volume, especially in terms of identifying and explaining causal forces. This is, one must add, an important distinguishing feature of the study; it does not attempt to provide a narrow, ordered, causally linked narrative of the way modernity and cinema acted on one another. Rather, it presents itself, appropriately described by Casetti himself, as a number of "glosses" which have an element of truth and authenticity, but which do not reduce the field of history to a single interpretation. Casetti intentionally leaves much unresolved, opening his argument to dialogical connections with other histories, conversations and glosses. This book does not offer final answers, or neatly ordered theoretical accounts, although the names of the chapters and concepts provide a certain structure. Such a complex cinema reality, Casetti seems to recognize, requires a discourse that does not necessarily dissolve every detail into simple, easy-to-understand descriptions. As Wittgenstein asked $(1967, \$ 77)$ in his consideration of the problems that arise in applying a reductive language to reality, "Won't it become a hopeless task to draw a sharp picture corre- 
sponding to a blurred one?" In the end, as with individual consciousness, which necessarily unites a wide range of stimulations into a single field of experience, so too does Casetti's performative approach bring together a broad range of glosses into a unified discursive field. This is perhaps, it must be said, Casetti's best virtue as a writer and intellectual; Eye of the Century is ultimately an exploration of complex human experiences, dispersed among a multitude of fragmented voices, sources and styles, yet told as an intriguing, intelligible and coherent story that contributes significant new knowledge to our understanding of the first decades of cinema, modernity and film.

Santiago Hidalgo

Université de Montréal

\section{BIBLIOGRAPHIC REFERENCES}

Wittgenstein 1967: Ludwig Wittgenstein, Philosophical Investigations [1953], 3rd ed., trans. G.E.M. Anscombe, Oxford, Blackwell, 1967. 